PROCEEDINGS OF THE

AMERICAN MATHEMATICAL SOCIETY

Volume 133, Number 9, Pages 2589-2596

S 0002-9939(05)08150-5

Article electronically published on April 19, 2005

\title{
COARSE EMBEDDINGS OF METRIC SPACES INTO BANACH SPACES
}

\author{
PIOTR W. NOWAK
}

(Communicated by Jonathan M. Borwein)

\begin{abstract}
There are several characterizations of coarse embeddability of locally finite metric spaces into a Hilbert space. In this note we give such characterizations for general metric spaces. By applying these results to the spaces $L_{p}(\mu)$, we get their coarse embeddability into a Hilbert space for $0<p<2$. This together with a theorem by Banach and Mazur yields that coarse embeddability into $\ell_{2}$ and into $L_{p}(0,1)$ are equivalent when $1 \leq p<2$. A theorem by $\mathrm{G}$.Yu and the above allow us to extend to $L_{p}(\mu), 0<p \leq 2$, the range of spaces, coarse embeddings into which is guaranteed for a finitely generated group $\Gamma$ to satisfy the Novikov Conjecture.
\end{abstract}

\section{INTRODUCTION}

Coarse embeddings were introduced by M. Gromov.

Definition 1.1 ([Gr, 7.E.]). Let $X, Y$ be metric spaces. A (not necessarily continuous function) $f: X \rightarrow Y$ is a coarse embedding if there exist non-decreasing functions $\rho_{1}, \rho_{2}:[0, \infty) \rightarrow[0, \infty)$ satisfying

(1) $\rho_{1}\left(d_{X}(x, y)\right) \leq d_{Y}(f(x), f(y)) \leq \rho_{2}\left(d_{X}(x, y)\right)$ for all $x, y \in X$,

(2) $\lim _{t \rightarrow \infty} \rho_{1}(t)=+\infty$.

In the language of coarse geometry, a coarse embedding $f: X \rightarrow Y$ is a coarse equivalence of $X$ and $f(X)$ (for the notions of coarse geometry we refer the reader to $\mathrm{Gr}$, Roe $)$.

Coarse embeddings have been in the spotlight recently due to a remarkable theorem by $\mathrm{G}$. $\mathrm{Yu}[\mathrm{Yu}$, who showed that every discrete metric space $\Gamma$ which embeds coarsely into a Hilbert space satisfies the Coarse Baum-Connes Conjecture. In particular, if $\Gamma$ is a finitely generated group with word length metric coarsely embeddable into a Hilbert space and the classifying space $B \Gamma$ has a homotopy type of a finite CW-complex, then the Novikov Conjecture holds for $\Gamma$.

Gromov asked [ $\mathrm{Gr}$ 7. $\left.\mathrm{E}_{2}\right]$ if every separable metric space embeds coarsely into a Hilbert space. A negative answer to this question was given by A.N. Dranishnikov, G. Gong, V. Lafforgue and G. Yu DGLY]. The counterexample was constructed using ideas of P. Enflo, who answered negatively a question raised by J. Smirnov whether every separable metric space is uniformly homeomorphic to a subset of a Hilbert space En.

Received by the editors October 5, 2003.

2000 Mathematics Subject Classification. Primary 46C05; Secondary 46 T99.

Key words and phrases. Coarse embeddings, metric spaces, Novikov Conjecture. 
Inspired by the work of Dranishnikov, Gong, Lafforgue and Yu [DGLY], we go somewhat further in this direction and try to find similarities between coarse embeddings and uniform embeddings (i.e., uniform homeomorphisms onto a subset). As a result, we generalize characterizations of coarse embeddability into $\ell_{2}$ given in [DGLY] in terms of negative definite functions from locally finite to general metric spaces. In particular we show that coarse embeddability of an arbitrary metric space depends only on its finite subsets. As an application we prove that for $0<p<2$ the space $L_{p}$ admits a coarse embedding into a Hilbert space. This and the fact that the space $L_{p}(0,1)$ for $p>1$ contains $\ell_{2}$ as a subspace yields that coarse embeddability into $\ell_{2}$ and into $L_{p}(0,1)$ are equivalent. All of the above combined with Yu's theorem on the Baum-Connes Conjecture $[\mathrm{Yu}]$ and the Descent Principle Roe implies obviously that the Novikov Conjecture holds for finitely generated groups coarsely embeddable into a separable $L_{p}(\mu)$-space, $0<p<2$.

Throughout this note we will talk about coarse embeddability, having in mind that the embedding is into a Hilbert space. We will also assume that all Hilbert spaces are real (this makes no loss to generality, since embedding into a complex Hilbert space is equivalent to embedding into a real Hilbert space).

Let us also note that if $f: X \rightarrow \mathcal{H}$ is a coarse embedding, then a reasonable assumption on $X$, allowing $\mathcal{H}=\ell_{2}$, is for $X$ to contain an at most countable $c$-net $\mathcal{C}$ for some constant $c>0$ (i.e., for every $x \in X$ there exists $y \in \mathcal{C}$ such that $d(x, y) \leq c)$. Of course, in the coarse category $\mathcal{C}$ and $X$ are isomorphic. In particular, this is the case when our metric space $X$ is a (finitely generated) group $\Gamma$ with a word length metric or (more generally) when $X$ is separable.

\section{Preliminaries}

Positive definite functions appeared in the work of E.H. Moore Mo. Let us briefly recall basic definitions and facts concerning positive and negative definite kernels and functions. For more details we refer the reader to [Sch, , BL, Chapter 8] and [AMM].

Definition 2.1. By a kernel on a set $X$ we mean any symmetric function $K: X \times X \rightarrow \mathbb{R}$. A kernel $K$ is said to be:

(1) positive definite if $\sum K\left(x_{i}, x_{j}\right) c_{i} c_{j} \geq 0$ for all $n \in \mathbb{N}$ and $x_{1}, \ldots, x_{n} \in X$, $c_{1}, \ldots, c_{n} \in \mathbb{R}$.

(2) negative definite if $\sum K\left(x_{i}, x_{j}\right) c_{i} c_{j} \leq 0$ for all $n \in \mathbb{N}$ and $x_{1}, \ldots, x_{n} \in X$, $c_{1}, \ldots, c_{n} \in \mathbb{R}$ such that $\sum c_{i}=0$.

A function $f: X \rightarrow \mathbb{R}$ on a commutative metric group $X$ is said to be positive (negative) definite if $K(x, y)=f(x-y)$ is a positive (negative) definite kernel.

A positive (negative) definite kernel $K$ is said to be normalized if $K(x, x)=1$ $(K(x, x)=0)$ for all $x \in X$. The set of positive definite kernels is closed under limits in the function space $\mathbb{R}^{X \times X}$ with pointwise convergence.

The following theorem describes the relation between negative and positive definite kernels.

Theorem 2.2 (I.J. Schoenberg $\left[\mathrm{Sch}_{2}\right.$; see also [BL, Proposition 8.4]). A kernel $N$ on $X$ is negative definite if and only if $e^{-t N}$ is positive definite for every $t>0$.

The key to study the connection between maps into Hilbert spaces and positive and negative definite kernels is the following theorem (see [BL, Proposition 8.5]). 
Theorem 2.3 ((1) E.H. Moore [Mo]; (2) I.J. Schoenberg [Sch $]$ ). (1) A kernel K on a set $X$ is positive definite if and only if there exist a Hilbert space $\mathcal{H}$ and a map $f: X \rightarrow \mathcal{H}$ such that

$$
K(x, y)=\langle f(x), f(y)\rangle \quad \text { for all } x, y \in X .
$$

(2) A normalized kernel $N$ on $X$ is negative definite if and only if there exist a Hilbert space $\mathcal{H}$ and a map $f: X \rightarrow \mathcal{H}$ such that

$$
N(x, y)=\|f(x)-f(y)\|^{2} \quad \text { for all } x, y \in X .
$$

Part (1) of Theorem 2.3 is the main tool in the study of uniform embeddings (i.e., uniform homeomorphisms onto a subset) into Hilbert spaces (see [AMM, BL]) while part (2) gives, as an immediate consequence, the well-known Schoenberg's characterization of metric spaces which embed isometrically into a Hilbert space: a metric space $(X, d)$ embeds isometrically into a Hilbert space if and only if $d^{2}$ is a negative definite kernel on $X$.

\section{Coarse embeddings into Hilbert spaces AND NEGATIVE DEFINITE KERNELS}

In this section we characterize coarse embeddability of metric spaces. In DGLY] the authors give a condition equivalent to coarse embeddability of a locally finite metric space into a Hilbert space in terms of negative definite kernels [DGLY, Theorem 2.2] (recall that a metric space is locally finite if every ball has finitely many elements). This characterization remains true for general metric spaces.

Proposition 3.1. A metric space $X$ admits a coarse embedding into a Hilbert space if and only if there exist a normalized, negative definite kernel on $X$ and non-decreasing functions $\rho_{i}:[0, \infty) \rightarrow[0, \infty), i=1,2$, satisfying

(1) $\rho_{1}(d(x, y)) \leq N(x, y) \leq \rho_{2}(d(x, y))$;

(2) $\lim _{t \rightarrow \infty} \rho_{1}(t)=\infty$.

Proof. Suppose that there exists a kernel $N$ satisfying conditions (1) and (2). By Theorem 2.3, there exist a Hilbert space $\mathcal{H}$ and a map $f: X \rightarrow \mathcal{H}$ such that

$$
N(x, y)=\|f(x)-f(y)\|^{2}
$$

for all $x, y \in X$. In other words, $f$ satisfies

$$
\sqrt{\rho_{1}(d(x, y))} \leq\|f(x)-f(y)\| \leq \sqrt{\rho_{2}(d(x, y))} .
$$

Obviously, $f$ is a coarse embedding.

Now suppose that $f: X \rightarrow \mathcal{H}$ is a coarse embedding and define $N(x, y)=$ $\|f(x)-f(y)\|^{2}$. Then, $N$ is a normalized, negative definite kernel on $X$ : when $\sum c_{i}=0$ and $x_{1}, \ldots, x_{n} \in X$, then

$$
\sum_{i, j=1}^{n}\left\|f\left(x_{i}\right)\right\|^{2} c_{i} c_{j}=\left(\sum_{i=1}^{n} c_{i}\right)\left(\sum_{j=1}^{n}\left\|f\left(x_{i}\right)\right\|^{2} c_{j}\right) ;
$$


hence

$$
\begin{aligned}
& \sum_{i, j=1}^{n} N\left(x_{i}, x_{j}\right) c_{i} c_{j} \\
&=\sum_{i, j=1}^{n}\left(\left\|f\left(x_{i}\right)\right\|^{2}+\left\|f\left(x_{j}\right)\right\|^{2}-2\left\langle f\left(x_{i}\right), f\left(x_{j}\right)\right\rangle\right) c_{i} c_{j} \\
&=-2\left\|\sum_{i=1}^{n} c_{i} f\left(x_{i}\right)\right\|^{2} \leq 0 .
\end{aligned}
$$

Remark 3.2. The proofs of Theorem 2.3 and Theorem 2.2 [DGLY use the same reasoning. In fact, the proof of the latter works also in the case of general metric spaces, except that for not locally finite spaces the construction may result in a non-separable Hilbert space.

Proposition 3.1 leads to the following.

Corollary 3.3. Let $(X, d)$ be a metric space. If for some non-decreasing function $\alpha:[0, \infty) \rightarrow[0, \infty)$ satisfying $\lim _{t \rightarrow \infty} \alpha(t)=\infty$ the kernel $\alpha \circ d$ is negative definite, then $X$ admits a coarse embedding into a Hilbert space.

Proof. If $\alpha \circ d$ is a negative kernel on $X$, then it satisfies the conditions of Proposition 3.1 with $\rho_{1}(t)=\rho_{2}(t)=\alpha(t)$.

Also in [DGLY] the authors prove that coarse embeddability in the case of locally finite metric spaces can be reduced to the question, whether all finite subsets can be mapped into a Hilbert space by functions having uniform estimates on the distance of images of two points [DGLY, Definition 3.1, Proposition 3.2].

We will show that again the local finiteness assumption can be dropped and that coarse embeddability of a metric space into a Hilbert space is determined by its finite subsets.

Theorem 3.4. A metric space $X$ admits a coarse embedding into a Hilbert space if and only if there exist non-decreasing functions $\rho_{i}:[0, \infty) \rightarrow[0, \infty), i=1,2$, such that $\lim _{t \rightarrow \infty} \rho_{1}(t)=\infty$ and for every finite subset $A \subset X$ there exists a map $f_{A}: A \rightarrow \ell_{2}$ satisfying

$$
\rho_{1}(d(x, y)) \leq\left\|f_{A}(x)-f_{A}(y)\right\| \leq \rho_{2}(d(x, y))
$$

for every $x, y \in X$.

Proof. Suppose that there exist functions $\rho_{1}, \rho_{2}$ and $f_{A}: A \rightarrow \ell_{2}$ as above. The kernel $N_{A}(x, y)=\left\|f_{A}(x)-f_{A}(y)\right\|^{2}$ is negative definite on $A$, and we have

$$
\rho_{1}^{2}(d(x, y)) \leq N_{A}(x, y) \leq \rho_{2}^{2}(d(x, y))
$$

for all $x, y \in A$. Define a new kernel $K_{A}=e^{-N_{A}}$. By Theorem 2.2 the kernel $K_{A}$ is positive definite and

$$
e^{-\rho_{2}^{2}(d(x, y))} \leq K_{A}(x, y) \leq e^{-\rho_{1}^{2}(d(x, y))} .
$$

Extend the kernel $K_{A}$ to a positive definite kernel $\widetilde{K}_{A}$ on $X$, by defining it to be 0 outside the set $A \times A$. For all $x, y \in X$ we have $\left|\widetilde{K}_{A}(x, y)\right| \leq 1$; thus the closure of the set $\left\{\widetilde{K}_{A}: \# A<\infty\right\}$ in the space $\mathbb{R}^{X \times X}$ with pointwise convergence topology 
is compact, by Tichonov's theorem. Let $K$ be a pointwise limit of a convergent subnet of the net $\left\{\widetilde{K}_{A}: \# A<\infty\right\}$. Then $K$ is a positive definite kernel on $X$, satisfying

$$
e^{-\rho_{2}^{2}(d(x, y))} \leq K(x, y) \leq e^{-\rho_{1}^{2}(d(x, y))}
$$

for all $x, y \in X$.

Since $K(x, y)>0$ for all $x, y \in X$, we can set $N(x, y)=-\ln K(x, y)$. We will show that $N$ is negative definite. Indeed, since $K$ is a pointwise limit and the function $e^{-x}$ is continuous, for every $t>0$ we have

$$
e^{-t N(x, y)}=K(x, y)^{t}=\left(\lim \widetilde{K}_{A}(x, y)\right)^{t}=\lim \left(\widetilde{K}_{A}(x, y)\right)^{t}
$$

where the limit is the one of the convergent subnet. By Theorem 2.2 the kernels $\widetilde{K}_{A}^{t}$ are positive definite for every $t>0$ and the right-hand side of equation (1) is a positive definite kernel as a pointwise limit of positive definite kernels. Thus $e^{-t N(x, y)}$ is positive definite for every $t>0$ and Theorem 2.2 yields that $N$ is a negative definite kernel on $X$ satisfying

$$
\rho_{1}^{2}(d(x, y)) \leq N(x, y) \leq \rho_{2}^{2}(d(x, y)) .
$$

By Proposition $3.1 X$ admits a coarse embedding into a Hilbert space.

The other implication is obvious.

\section{Coarse embeddings of $L_{p}$-SPaces}

In this section, as an application of the above results, we show that the space $L_{p}(\mu)$ is coarsely embeddable for $0<p \leq 2$, which gives, as an immediate consequence, a stronger version of [DGLY, Proposition 3.3], where the authors showed that every locally finite subspace of $\ell_{1}(\mathbb{N})$ admits a coarse embedding into a Hilbert space.

The spaces $L_{p}(\mu)$ are metric only for $p \geq 1$, but we will consider also the case $0<p<1$.

Proposition 4.1. For any measure $\mu$, the space $L_{p}(\mu)$ (in particular $\ell_{p}$ ) admits a coarse embedding into a Hilbert space for $0<p<2$.

To prove Proposition 4.1 we need the following lemma, which was first proved by I.J. Schoenberg $\left[\mathrm{Sch}_{1}\right.$, Theorem 2].

Lemma 4.2. Let $N$ be a negative definite kernel on $X$ and $N(x, y) \geq 0$ for all $x, y \in X$. Then the kernel $N^{\alpha}$ is negative definite for any $0<\alpha<1$.

Proof of the lemma. Let $N$ be a negative definite kernel. Then for every $t \geq 0$ the kernel $1-e^{-t N} \geq 0$ is also negative definite, and we have

$$
\int_{0}^{\infty}\left(1-e^{-t N}\right) d \mu(t) \geq 0
$$

for every positive measure $\mu$ on $[0, \infty)$. For every $x>0$ and $0<\alpha<1$ the following formula holds:

$$
x^{\alpha}=c_{\alpha} \int_{0}^{\infty}\left(1-e^{-t x}\right) t^{-\alpha-1} d t,
$$

\footnotetext{
${ }^{1}$ Here, by a net we mean a generalized sequence.
} 
where $c_{\alpha}$ is some positive constant. Thus $N^{\alpha}$ is also a negative definite kernel for every $0<\alpha<1$.

Proof of Proposition 4.1. The kernel $|x-y|^{2}$ is negative definite on the real line (as a square of the metric on a Hilbert space). By the above lemma, for any $0<p \leq 2$ the kernel $|x-y|^{p}$ also is negative definite on $\mathbb{R}$, i.e.,

$$
\sum\left|x_{i}-x_{j}\right|^{p} c_{i} c_{j} \leq 0
$$

for every such $p$, all $x_{1}, \ldots, x_{n} \in \mathbb{R}$ and $c_{1}, \ldots, c_{n} \in \mathbb{R}$ such that $\sum c_{i}=0$. By integrating the above inequality with respect to the measure $\mu$, we get that the function $\|x\|^{p}$ is negative definite on $L_{p}(\mu)$ and the assertion follows from Corollary 3.3 .

Corollary 4.3. If a metric space $X$ admits a coarse embedding into the space $L_{p}(\mu)$ for $0<p<2$ and some measure $\mu$ (in particular into $\ell_{p}$ ), then $X$ admits a coarse embedding into a Hilbert space.

G.Yu showed that every discrete metric space $\Gamma$ with bounded geometry which admits a coarse embedding into a Hilbert space satisfies the Coarse Baum-Connes Conjecture $\mathrm{Yu}$. In particular, if $\Gamma$ is a finitely generated group with word length metric, then the Descent Principle Roe] implies that the Novikov Conjecture holds for $\Gamma$. To situate the results of this section in the context of the Novikov Conjecture we give this obvious

Corollary 4.4. Let $\Gamma$ be a finitely generated group and let the classifying space $B \Gamma$ have the homotopy type of a finite $C W$-complex. If $\Gamma$ (as a metric space with word length metric) admits a coarse embedding into a (separable) space $L_{p}(\mu), 0<p<2$, then the Novikov Conjecture holds for $\Gamma$.

Let us also note an interesting consequence of a theorem due to S. Banach and S. Mazur on the linear dimension of the spaces $L_{p}$ [Ba, Theorem 6, p.123] which states that $\ell_{2}$ is isomorphic to a subspace $L_{p}(0,1)$ if $p>1$. From this we conclude that every separable metric space, coarsely embeddable into a Hilbert space, admits a coarse embedding into any $L_{p}(0,1)$ with $p \geq 1$. Moreover, for $1<p \leq 2$ the space $L_{p}(0,1)$ is isometric to a subspace of $L_{1}(0,1)$ ([LT, Theorem II.3.14, p.139]). Thus, we arrive at the following.

Corollary 4.5. Let $X$ be a separable metric space. The following statements are equivalent:

(1) $X$ is coarsely embeddable into $\ell_{2}$;

(2) $X$ is coarsely embeddable into $L_{p}(0,1)$ for some (equivalently all) $1 \leq p<2$.

\section{Final REMARKS}

(A) Let $\mathcal{C}$ denote the set of all integer sequences which are eventually zero. This is the abelian group $\bigoplus_{n \in \mathbb{N}} \mathbb{Z}$ on which the word length metric (with respect to the standard generators) is just the $\ell_{1}$ metric. With this metric $\mathcal{C}$ is coarsely embeddable into $\ell_{2}$ by Proposition 4.1. There is another natural metric on $\mathcal{C}$, induced by the sup-norm $\|\cdot\|_{0}$. We claim that $\left(\mathcal{C},\|\cdot\|_{0}\right)$ is not coarsely embeddable into $\ell_{2}$.

To show this consider $\left(\mathcal{C},\|\cdot\|_{0}\right)$ as a 1 -net in the space $c_{0}$. If $\left(\mathcal{C},\|\cdot\|_{0}\right)$ would be coarsely embeddable into $\ell_{2}$, then so would be $c_{0}$. By a result of I. Aharoni Ah 
there exists a constant $K>0$ such that for every separable metric space $X$ there is a map $T: X \rightarrow c_{0}$ satisfying

$$
d(x, y) \leq\|T x-T y\| \leq K d(x, y) \quad \text { for all } x, y \in X .
$$

Therefore any separable metric space would be coarsely embeddable into $\ell_{2}$, which is not the case by a result in [DGLY]. This contradiction completes the proof.

Let us note that, unfortunately, the group $\mathcal{C}$ is infinitely generated and fails to be locally finite.

(B) The results in Section 4 are counterparts of facts from the theory of "classical" uniform embeddings into Hilbert space (see [AMM, BL]). A theorem of I. Aharoni, B. Maurey and B.S. Mityagin [AMM, Corollary 4.3] states that $L_{p}(\mu)$ is not uniformly homeomorphic to any subset of a Hilbert space if $p>2$. It is natural to ask, whether this result has a counterpart for coarse uniform embeddings, i.e., does the space $L_{p}(\mu)$ admit a coarse uniform embedding into a Hilbert space if $p>2$ ?

(C) Proposition 3.1 can provide an alternative proof of the characterization of coarse embeddability, given by M. Dadarlat and E. Guentner [DG, Proposition 2.1]: a metric space $X$ is coarsely embeddable into a Hilbert space $\mathcal{H}$ if and only if for every $R>0$ and every $\varepsilon>0$ there exist $S>0$ and a map $f: X \rightarrow \mathcal{H}$ such that for all $x, y \in X$ we have $\|f(x)\|=1, \sup \{\|f(x)-f(y)\|: d(x, y) \leq R\} \leq \varepsilon$ and $\lim _{S \rightarrow \infty} \inf \{\|f(x)-f(y)\|: d(x, y) \geq S\}=\sqrt{2}$. We leave the details to the reader.

(D) Since $c_{0}^{*}=\ell_{1}$ and $\ell_{1}^{*}=\ell_{\infty} \supset c_{0}$, Proposition 4.1 and the above discussion provide an example of a Banach space, which is coarsely embeddable, while its dual is not, and of a Banach space, which is not coarsely embeddable, while its dual is.

\section{ACKNOWLEDGEMENTS}

This note is a part of the author's M.Sc. thesis, written under the supervision of Prof. Henryk Toruńczyk at the Institute of Mathematics of the Warsaw University in Poland and defended in June, 2003.

The author wishes to express his gratitude to Prof. Toruńczyk for guidance and an uncountable number of valuable suggestions and remarks.

The author is also very grateful to Prof. Tadeusz Koźniewski and Prof. Zbigniew Marciniak for their active interest in the publication of this paper and to Prof. Guoliang Yu for encouragement.

\section{REFERENCES}

[Ah] I. Aharoni, Every separable metric space is Lipschitz equivalent to a subset of $c_{0}$, Isr. J. Math. 19 (1974) 284-291. MR0511661 (58:23471a)

[AMM] I. Aharoni, B. Maurey, B. S. Mityagin, Uniform embeddings of metric spaces and of Banach spaces into Hilbert Spaces, Isr. J. Math., 52 (1985), 251-265. MR0815815 (87b:46011)

[Ba] S. BANACH, Theory of Linear Operations, North-Holland Mathematical Library, Volume 38 (1987). MR0880204 (88a:01065)

[BL] Y. Benyamini, J. Lindenstrauss, Geometric nonlinear functional anlysis, Volume 48 of Colloquium Publications. American Mathematical Society, Providence, R.I., 2000. MR1727673 (2001b:46001)

[DG] M. DAdARlat, E. Guentner, Constructions preserving Hilbert space uniform embeddability of discrete groups, Trans. Amer. Math. Soc. 355 (2003), 3253-3275. MR1974686 (2004e:20070) 
[DGLY] A.N. Dranishnikov, G. Gong, V. Lafforgue, G. Yu, Uniform Embeddings into Hilbert Space and a Question of Gromov, Canad. Math. Bull., Vol.45(1), 2002, 60-70. MR1884134 (2003a:57043)

[En] P. Enflo, On a problem of Smirnov, Ark. Math. 8 (1969), 107-109. MR0415576 $(54: 3661)$

[Gr] M. Gromov, Asymptotic invariants of infinite groups, London Mathematical Society Lecture Notes, no.182, s. 1-295, Cambridge University Press, 1993. MR1253544 (95m:20041)

[LT] J. Lindenstrauss, L. Tzafriri, Classical Banach spaces, Springer-Verlag Lecture Notes in Mathematics 338 (1973). MR0415253 (54:3344)

[Mo] E.H. Moore, On properly positive Hermitian matrices, Bull. Am. Math. Soc. 23 (1916), $59,66-67$.

[Roe] J. RoE, Index theory, coarse geometry, and topology of manifolds, CBMS Regional Conference Series in Mathematics, AMS 1996. MR1399087 (97h:58155)

[Sch 1 I.J. SchoenberG, On certain metric spaces arising from euclidean spaces by a change of metric and their imbedding in Hilbert space, Ann. Math. 38 (1937), 787-793. MR1503370

[Sch 2$]$ I.J. SchoenberG, Metric spaces and positive definite functions, Trans. Am. Math. Soc. 44 (1938), 522-536. MR1501980

[Yu G. Yu, The coarse Baum-Connes conjecture for spaces which admit a uniform embedding into Hilbert space, Invent. Math. (1) 139 (2000), 201-240. MR1728880 (2000j:19005)

Institute of Mathematics, Warsaw University, ul. Banacha 2, 02-097 Warsaw, Poland - And - Department of Mathematics, Tulane University, 6823 St. Charles Avenue, New ORLEANS, LOUISIANA 70118

Current address: Department of Mathematics, Vanderbilt University, 1326 Stevenson Center, Nashville, Tennessee 37240

E-mail address: pnowak@math.vanderbilt.edu 